\title{
Higher rate of colon polyp detection aided by an artificial intelligent software
}

\author{
Masaaki Iwatsuki ${ }^{1,2}$, Kazuto Harada ${ }^{1,2}$, Hideo Baba $^{2}$, Jaffer A. Ajani ${ }^{1}$ \\ ${ }^{1}$ Department of Gastrointestinal Medical Oncology, The University of Texas MD Anderson Cancer Center, Houston, TX, USA; ${ }^{2}$ Department of \\ Gastroenterological Surgery, Graduate School of Medical Sciences, Kumamoto University, Kumamoto, Japan \\ Correspondence to: Jaffer A. Ajani. Department of Gastrointestinal Medical Oncology, The University of Texas MD Anderson Cancer Center, 1515 \\ Holcombe Blvd, Houston, TX 77030, USA. Email: jajani@mdanderson.org. \\ Provenance: This is an invited Editorial commissioned by Section Editor Qiang Shi (Zhongshan Hospital, Fudan University, Shanghai, China). \\ Comment on: Urban G, Tripathi P, Alkayali T, et al. Deep Learning Localizes and Identifies Polyps in Real Time With 96\% Accuracy in Screening \\ Colonoscopy. Gastroenterology 2018;155:1069-78.e8.
}

Received: 30 November 2018; Accepted: 06 December 2018; Published: 24 December 2018.

doi: $10.21037 /$ tgh.2018.12.05

View this article at: http://dx.doi.org/10.21037/tgh.2018.12.05

Colorectal cancer (CRC) is one of the most common malignancies in the United States and Europe $(1,2)$. Detection and removal of colon polyps or early cancer is associated with a reduction in mortality from CRC (3). The adenoma detection rate (ADR) during screening colonoscopy is recommended as a measure of the quality of colonoscopic examination. Corley et al. reveled that a $1 \%$ increase in the $\mathrm{ADR}$ was associated with a $3 \%$ decrease in interval CRC incidence (4). More recently, a prospective study from Poland et al. showed $1 \%$ increase in the ADR was associated with a $6 \%$ reduced risk of interval CRC (5). However, the ADR varies widely, largely depending on the ability of gastroenterologist, time spent, and preparation quality. Unfortunately, adenoma miss rate remains high (6-27\%) (6), despite of novel technologies, devises, and interpretation. It is partly because of well recognized blind spots on colonoscopies.

To resolve these issues, artificial intelligence (AI) has been applied to the field of gastrointestinal endoscopy. Since 1990s, the method for computer-aided detection (CADe) for colorectal polyps has been investigated combining texture, color or mixed analysis. Finally, CADe method with higher sensitivity is firstly reported by Karkanis et al., which achieved $>90 \%$ detection rate (7). However, these systems have not been widely prevalent in the clinical setting because these systems were established base on the static endoscopic image. Therefore, focus of research in this field shifted to the real-time imaging analysis. In 2016, real-time colonic polyp detection systems were developed, which have the challenge of having low sensitivity due to traditional machine learning methods (8). These problems were dramatically overcome through the deep learning method into CADe system. Recently, Misawa et al. developed a CADe system for colorectal polyps based on deep learning method (9). This system was trained by a total 546 short videos including 155 polyp-positive and 391 polyp-negative short videos. These videos were randomly divided into 2 groups: learning group (105 positive and 306 negative) and test group (50 positive and 85 negative polyp). This CADe system detected $94 \%$ (47 of 50 ) of the test group with $60 \%$ false-positive detection (51 of 85).

Most recently, Urban et al. built a novel CADe system in real time with $96 \%$ accuracy reported in the journal Gastroenterology (10). They designed CAD system using convolutional neural networks (CNN called "Deep Learning") in a set of 8,641 hand-labeled images containing 4,008 unique polyps collected from more than 2,000 patients. To achieve widespread clinical use, accuracy, portability, and rapid processing speed are desired. Therefore, this study was carefully designated by following several datasets, which were used to train and evaluate the deep learning model, separately polypdetection and localization. (I) The ImageNet challenge dataset (1.2 million natural images); (II) 8,641 handselected colonoscopy images (4,088 unique polyps and 4,553 images without polyps), including white light and 
NBI images; (III) a separate 1,330 colonoscopy images collected from different patients (672 unique polyp and 658 non-polyp images); (IV) and 9 colonoscopy videos; (V) a combined dataset consisting original 8,641 images and additional 44,947 images were extracted from 9 videos; (VI) additionally, 11 "challenging" colonoscopy videos performed by highly skilled endoscopists ( $\mathrm{ADR} \geq 50 \%$ ) were used. For polyp detection, a test of accuracy of the CNN trained on the 8,641 images was $96.4 \%$ and the ROC-AUC of 0.794 in the independent 1,330 images dataset. In the analysis of colonoscopy video, 36 polyps were identified by 3 endoscopy experts and 45 polyps were identified by the CNN system (false-positive rate; 7\%).

In this study, some interesting additional experiments were performed. First question was: can CNN classify all polyps in spite of its morphology? Nonpolypoid lesion (flat and depressed) were often not recognized by even skilled endoscopists compared with polypoid lesion. The CNN system missed 12\% (84 of 678) polypoid polyps (Ip and Is) and $11 \%$ (41 of 381) nonpolypoid polyps (IIa, IIb and IIc). Therefore, polyp morphology does not impact on the performance of CNN system. Second question asked was: can $\mathrm{CNN}$ detect polyps under conditions of hurried withdrawal or poor inspection? With the use of purposefully difficult 11 colonoscopy videos, featuring "flyby" scenarios without closing to previously found polyps during withdrawal, missed polyps were located in "flyby" segments of the video. Therefore, $\mathrm{CNN}$ is less attuned to quick movement by endoscopists at this stage. Third question asked was: is it beneficial to train the CNN on NBI plus white light images? Recently, a CNN system trained by white light and NBI endoscopy images was developed, which achieved a 94\% accuracy in differentiating diminutive adenomas from hyperplastic polyps (11). Although NBI or chromoendoscopy is very useful modality to characterize small polyps during screening or surveillance colonoscopy, inter- or intra-observer variability are unavoidable problems, depending on endoscopist's ability. Such computer-aided diagnosis (CADx) may decrease variance among endoscopists.

The works by Urban et al. has a number of prospective applications on the screening or surveillance colonoscopy. We congratulate them on their fabulous effort to move this field forward. Their willingness to loan the technology without asking for royalties and to benefit the population in general rather than subscribe to profit taking mentality.

In the future, more integration of the CADe and CADx systems may enable us to detect polyps more precisely and speedily in the clinical setting. However, transportability and acceptance of this technology waits for now...

\section{Acknowledgements}

Funding: This work was supported in part by a Grantin-Aid for Scientific Research from the Japan Society for the Promotion of Science, grant number 16K10463 and $16 \mathrm{KK} 0184$

\section{Footnote}

Conflicts of Interest: The authors have no conflicts of interest to declare.

\section{References}

1. Ferlay J, Colombet M, Soerjomataram I, et al. Cancer incidence and mortality patterns in Europe: Estimates for 40 countries and 25 major cancers in 2018. Eur J Cancer 2018;103:356-387.

2. Siegel RL, Miller KD, Fedewa SA, et al. Colorectal cancer statistics, 2017. CA Cancer J Clin 2017;67:177-93.

3. Zauber AG, Winawer SJ, O'Brien MJ, et al. Colonoscopic polypectomy and long-term prevention of colorectalcancer deaths. N Engl J Med 2012;366:687-96.

4. Corley DA, Jensen CD, Marks AR, et al. Adenoma detection rate and risk of colorectal cancer and death. $\mathrm{N}$ Engl J Med. 2014;370:1298-306.

5. Kaminski MF, Wieszczy P, Rupinski M, et al. Increased Rate of Adenoma Detection Associates With Reduced Risk of Colorectal Cancer and Death. Gastroenterology 2017;153:98-105.

6. Ahn SB, Han DS, Bae JH, et al. The Miss Rate for Colorectal Adenoma Determined by Quality-Adjusted, Back-to-Back Colonoscopies. Gut Liver 2012;6:64-70.

7. Karkanis SA, Iakovidis DK, Maroulis DE, et al. Computeraided tumor detection in endoscopic video using color wavelet features. IEEE Trans Inf Technol Biomed 2003;7:141-52.

8. Fernández-Esparrach G, Bernal J, López-Cerón M, et al. Exploring the clinical potential of an automatic colonic polyp detection method based on the creation of energy maps. Endoscopy 2016;48:837-42.

9. Misawa M, Kudo SE, Mori Y, et al. Characterization of Colorectal Lesions Using a Computer-Aided Diagnostic System for Narrow-Band Imaging Endocytoscopy. Gastroenterology 2016;150:1531-2.e3. 
10. Urban G, Tripathi P, Alkayali T, et al. Deep Learning Localizes and Identifies Polyps in Real Time With 96\% Accuracy in Screening Colonoscopy. Gastroenterology 2018;155:1069-78.e8.

11. Byrne MF, Chapados N, Soudan F, et al. Real-time

doi: $10.21037 / \operatorname{tgh} .2018 .12 .05$

Cite this article as: Iwatsuki M, Harada K, Baba H, Ajani JA. Higher rate of colon polyp detection aided by an artificial intelligent software. Transl Gastroenterol Hepatol 2018;3:106. differentiation of adenomatous and hyperplastic diminutive colorectal polyps during analysis of unaltered videos of standard colonoscopy using a deep learning model. Gut 2019;68:94-100. 